\title{
Visualization \& Prediction of COVID-19 Future Outbreak by Using Machine Learning
}

\author{
Ahmed Hassan Mohammed Hassan \\ Faculty of Engineering, Mashreq University, Khartoum North, Sudan \\ E-mail: dr.ahmed@mashreq.edu.sd

\section{Arfan Ali Mohammed Qasem} \\ Faculty of Engineering, Mashreq University, Khartoum North, Sudan \\ E-mail: basealali@gmail.com \\ Walaa Faisal Mohammed Abdalla ${ }^{1}$ and Omer H. Elhassan ${ }^{2}$ \\ ${ }^{1}$ Faculty of Engineering, Mashreq University, Khartoum North, Sudan \\ ${ }^{2}$ Mashreq University, Khartoum North, Sudan \\ E-mail: ${ }^{1}$ walaafaisal123@gmail.com, ${ }^{2}$ president@mashreq.edu.sd
}

Received: 25 October 2020; Accepted: 03 April 2021; Published: 08 June 2021

\begin{abstract}
Day by day, the accumulative incidence of COVID-19 is rapidly increasing. After the spread of the Corona epidemic and the death of more than a million people around the world countries, scientists and researchers have tended to conduct research and take advantage of modern technologies to learn machine to help the world to get rid of the Coronavirus (COVID-19) epidemic. To track and predict the disease Machine Learning (ML) can be deployed very effectively. ML techniques have been anticipated in areas that need to identify dangerous negative factors and define their priorities. The significance of a proposed system is to find the predict the number of people infected with COVID19 using ML. Four standard models anticipate COVID-19 prediction, which are Neural Network (NN), Support Vector Machines (SVM), Bayesian Network (BN) and Polynomial Regression (PR). The data utilized to test these models content of number of deaths, newly infected cases, and recoveries in the next 20 days. Five measures parameters were used to evaluate the performance of each model, namely root mean squared error (RMSE), mean squared error (MAE), mean absolute error (MSE), Explained Variance score and $\mathrm{r} 2$ score $\left(\mathrm{R}^{2}\right)$. The significance and value of proposed system auspicious mechanism to anticipate these models for the current scenario of the COVID-19 epidemic. The results showed NN outperformed the other models, while in the available dataset the SVM performs poorly in all the prediction. Reference to our results showed that injuries will increase slightly in the coming days. Also, we find that the results give rise to hope due to the low death rate. For future perspective, case explanation and data amalgamation must be kept up persistently.
\end{abstract}

Index Terms: COVID-19, Deep Neural Network, Support Vector Machines, Bayesian Network, Polynomial Regression Pandemic.

\section{Introduction}

There are many epidemics that have invaded humanity throughout history. In December 2019, a disease appeared in Wuhan, China, this disease spread very quickly around the world and countries could not control it, prompting the World Health Organization in March 2020 to declare the COVID-19 epidemic is a global pandemic [1]. The new COVID-19 fulminate in more than 186 countries infecting cases 34.2 million individuals and causing 1.02 million deaths by OCT 02, 2020. COVID 19 causes many effects on the human body, such as influenza symptoms, failure in many organs, and acute respiratory syndrome, which may eventually lead to the death of people, especially the elderly and Patient with chronic diseases [2].

ML has imposed itself as a science capable of solving very complex real-world problems of past years. Machine learning and artificial intelligence have been used in most areas of life such as medicine [3], autonomous cars, robotics, weather forecasting [4], image processing, natural language, [5], UAV,.. etc. [6]. ML algorithms surpass traditional algorithms in that they use the method of learning from experiences and correcting errors based on these experiences as a human being. Prediction is one of the most important areas of ML. ML has been used to predict diseases.

The major objectives of this research to contribute to getting rid of the virus and help save humanity. We will develop a predictive model that predicts the spread of the Coronavirus using ML. Our methodology consider three cases 
predicted within the next 20 days, the number of confirmed, deaths, and recovery cases. The ML algorithms used are NN, SVM, Bayesian Network, PR [7, 8]. The ML models used in this study are trained using a set of statistical data for daily cases of COVID-19 patients provided by a which are continuously updated. This data is processed and divided into a training data set $(85 \%)$ and a test data set $(15 \%)$.

In the absence of any medical advices, the only solution to stop the spread by training social distancing and cleanliness. This up normal of behavior of COVID-19 requires evolving method for tracking its prevalence and automation of the tracking tools for dynamic decision making.

We know that the ML can be utilized to handle large data and intelligently predict the next step of any model such as spreading any dangers disease. The Motivation and our contributions of this paper has findings the prediction model using ML algorithms can be profitable for decision-makers to restrain COVID-19 pandemics. This manuscript, provide a prediction model deployed using for precise prediction of the number of COVID-19 cases: confirmed, deaths and recoveries in near future. Furthermore, provide a detailed of model comparison based on MSE, RMSE, MAE and R ${ }^{2}$ parameters. Finally, we summarize this work and present various research directions.

\section{Related Work}

There are many studies conducted to predict future diseases using regression and artificial intelligence networks. ML techniques have been used to predict chronic diseases [9], diabetes [10], heart disease [11], Breast Cancer [12] and other diseases to help the doctor make the right decision.

COVID 19 spreads very quickly between people, through air or physical contact, or by touching surfaces contaminated with the virus, so it poses a great danger to humanity [13, 14]. Doctors, scientists, and researchers in various parts of the world are making efforts to provide the appropriate treatment and vaccine to get rid of COVID-19. Unfortunately, now there is no suitable vaccine for this disease. Limiting the spread of the virus in the coming days is very important, and therefore the governments of the world have taken strict measures such as closing airports and imposing a complete or partial ban. Technology helps limit the spread of the virus by predicting new cases [15]. ML algorithms are evaluated with the following measures parameters MSE, RMSE, MAE and R ${ }^{2}$ [16].

\section{Materials and Methods}

ML and Data Science community are hardly work to improve the estimates of epidemiological models and analyze the generation information. This article proposes a ML model that can be run continuously on Python platform to obtain the most accurate result of the development of the spread of the epidemic and to develop a strategy to avoid the spreading by the government and the citizen. Prediction of COVID-19 using ML in Python language is a technique implemented with the help of processing tool following:

- Jupyter Notebook environment: It is used to generate a single web document by merging executable code, text, formulas into. This is useful for many purposes such as debug, and so forth.

- library NumPy: Foundation library used for scientific computing in Python since. It offers data structures and high-performing purposes. It defines a precise data structure that is an N-dimensional array defined as ndarray. This library utilized in this article for element-wise computation and Reading-writing datasets.

- Pandas: this package is core of data analysis in Python and provides complex data structures to make the work easy, fast, and effective. Its work with NumPy library to manipulation of data in spreadsheets or in relational databases (SQL databases).

- Matplotlib: it used to generate the plots and other data visualizations in 2D.

Data Set: In this paper, COVID-19 dispersal predicts in the future using ML algorithms to taking into consideration the number of confirmed cases, recoveries and deaths. The ML models were constructed using the datasets from the GitHub repository provided by the Center for Systems Science and Engineering, Johns Hopkins University [17]. Datasets are included in the folder on the GitHub under the name (csse_covid_19_time_series). These Dataset files consist of daily time series tables, inclusive the number of recoveries cases, deaths cases, and confirmed cases. All data are from the daily case report and the update frequency of data is one day. Table 1, Table 2 and Table 3 are displayed the country, latitude, longitude and interval range (1/22/2020 to 04/10/2020) for recovery, death and new confirmed, respectively. The selection of MATERIALS AND METHODS facilitates to achieve research objectives was the assistance of experts in the field of ML and scientific papers. 
Table 1. Sample data of new recovery cases worldwide

\begin{tabular}{|c|c|c|c|c|c|c|c|c|c|c|c|}
\hline & $\begin{array}{l}\text { COUNTRY/ } \\
\text { REGION }\end{array}$ & LAT & LONG & $\begin{array}{c}1 / 22 / 2 \\
020\end{array}$ & $\begin{array}{l}1 / 23 / \\
2020\end{array}$ & $\cdots$ & $\begin{array}{l}9 / 30 / \\
2020\end{array}$ & $\begin{array}{l}10 / 1 / \\
2020\end{array}$ & $\begin{array}{l}10 / 2 / \\
2020\end{array}$ & $\begin{array}{l}10 / 3 / \\
2020\end{array}$ & $\begin{array}{l}10 / 4 / \\
2020\end{array}$ \\
\hline $\mathbf{0}$ & Afghanistan & 33.93911 & 67.709953 & 0 & 0 & $\ldots$ & 32789 & 32842 & 32842 & 32842 & 32852 \\
\hline 1 & Albania & 41.1533 & 20.1683 & 0 & 0 & $\ldots$ & 7847 & 8077 & 8342 & 8536 & 8675 \\
\hline 2 & Algeria & 28.0339 & 1.6596 & 0 & 0 & $\ldots$ & 36174 & 36282 & 36385 & 36482 & 36578 \\
\hline 3 & Andorra & 42.5063 & 1.5218 & 0 & 0 & $\ldots$ & 1432 & 1432 & 1540 & 1540 & 1540 \\
\hline 4 & Angola & -11.2027 & 17.8739 & 0 & 0 & $\ldots$ & 1941 & 2082 & 2215 & 2436 & 2577 \\
\hline
\end{tabular}

Table 2. Sample data of New death cases in worldwide

\begin{tabular}{c|ccccccccccc} 
& $\begin{array}{c}\text { COUNTRY } \\
\text { /REGION }\end{array}$ & LAT & LONG & $\begin{array}{c}\mathbf{1 / 2 2} / \mathbf{2} \\
\mathbf{0 2 0}\end{array}$ & $\begin{array}{c}\mathbf{1 / 2 3} / \mathbf{0} \\
\mathbf{0 2 0}\end{array}$ & $\mathbf{\cdots}$ & $\mathbf{2 0 2 0}$ & $\mathbf{2 0 2 0}$ & $\mathbf{2 0 2 0}$ & $\mathbf{2 0 2 0}$ & $\mathbf{2 0 2 0}$ \\
\hline $\mathbf{0}$ & Afghanistan & 33.93911 & 67.70995 & 0 & 0 & $\ldots$ & 1458 & 1458 & 1458 & 1462 & 1462 \\
$\mathbf{1}$ & Albania & 41.1533 & 20.1683 & 0 & 0 & $\ldots$ & 387 & 388 & 389 & 392 & 396 \\
$\mathbf{2}$ & Algeria & 28.0339 & 1.6596 & 0 & 0 & $\ldots$ & 1736 & 1741 & 1749 & 1756 & 1760 \\
$\mathbf{3}$ & Andorra & 42.5063 & 1.5218 & 0 & 0 & $\ldots$ & 53 & 53 & 53 & 53 & 53 \\
$\mathbf{4}$ & Angola & -11.2027 & 17.8739 & 0 & 0 & $\ldots$ & 183 & 185 & 189 & 193 & 195
\end{tabular}

Table 3. Sample data of New Confirmed Cases in Worldwide

\begin{tabular}{|c|c|c|c|c|c|c|c|c|c|c|}
\hline & COUNTRY /REGION & LAT & LONG & $\begin{array}{l}1 / 22 / \\
2020\end{array}$ & $\cdots$ & $\begin{array}{c}9 / 30 \\
/ 2020\end{array}$ & $\begin{array}{c}10 / 1 \\
/ 2020\end{array}$ & $\begin{array}{c}10 / 2 \\
/ 2020\end{array}$ & $\begin{array}{c}10 / 3 \\
/ 2020\end{array}$ & $\begin{array}{c}10 / 4 \\
/ 2020\end{array}$ \\
\hline $\mathbf{0}$ & Afghanistan & 33.93911 & 67.709953 & 0 & $\ldots$ & 39268 & 39285 & 39290 & 39297 & 39341 \\
\hline $\mathbf{1}$ & Albania & 41.1533 & 20.1683 & 0 & $\ldots$ & 13649 & 13806 & 13965 & 14117 & 14266 \\
\hline 2 & Algeria & 28.0339 & 1.6596 & 0 & $\ldots$ & 51530 & 51690 & 51847 & 51995 & 52136 \\
\hline 3 & Andorra & 42.5063 & 1.5218 & 0 & $\ldots$ & 2050 & 2050 & 2110 & 2110 & 2110 \\
\hline 4 & Angola & -11.2027 & 17.8739 & 0 & $\ldots$ & 4972 & 5114 & 5211 & 5370 & 5402 \\
\hline
\end{tabular}

\section{PREDICTION MODEL:}

Four regression models have been used to predict the COVID-19: NN, SVM, BR and PR. In this paper, to denote the NN we used the ML Perceptron regressor (MLP) [18]. MLP model using LBFGS or stochastic gradient descent to optimizes the squared-loss. MLP regressor trains iteratively since at each time step the partial derivatives of the loss function with respect to the model parameters are computed to update the parameters. To update the parameters of MLP always use concept of iterative to obtain the partial derivatives of the loss function with respect to parameters of the model to compute the new value of parameters [19-22]. In our model, we used parameters as follow:

a) in case of recoveries

alpha $=0.0001$, hidden layer sizes $=(30,30)$, random state $=35$, learning rate $={ }^{\prime}$ constant, solver $=' l b f g s^{\prime}$, activation='relu'.

b) in case of confirmed

alpha $=0.001$, hidden layer sizes $=(100,60)$, random state $=25$, learning rate $={ }^{\prime}$ constant, solver $=' l b f g s^{\prime}$, activation='relu'.

c) in case of deaths

alpha $=0.001$, hidden layer sizes $=(500,120)$, random state $=25$, learning rate $=$ 'constant, solver $=$ 'adaptive', activation='relu'.

Secondly, SVM [23] use a mechanism called kernels. This mechanism converts the input data into the required output. SVM solves the regression problems using a linear function but in our case we map the input $(\mathrm{x})$ to a feature space (z) (i.e, n-dimensional space) because our it has non-linearity behavior. In this paper, we used parameter as follow:

a) in case of recoveries

shrinking $=$ True, kernel $={ }^{\prime}$ poly', gamma $=0.01$, epsilon $=1$, degree $=4, C=1$

b) in case of confirmed

$\left(\right.$ shrinking $=$ False, kernel $={ }^{\prime}$ poly', gamma $=0.01$,epsilon $=0.01$,degree $\left.=3, C=0.1\right)$

c) in case of deaths

(shrinking $=$ True, kernel $={ }^{\prime}$ poly', gamma $=0.1$, epsilon $=0.01$, degree $\left.=2, C=0.01\right)$ 
Thirdly, the utilization of Bayesian inference [24] was used to construct the models where the update of the parameters using Sparse Bayesian Learning and the Relevance Vector Machine [25]. When talking about hierarchical data structure the Bayesian modeling framework has been praised for its capability to deal with it [26].

Furthermore, Polynomial Regression is a form of regression analysis in which the relationship between the independent variable $(\mathrm{x})$ and the dependent variable $(\mathrm{y})$ are modeled as $\left(\mathrm{n}^{\text {th }}\right)$ degree polynomial in $(\mathrm{x})$.

\section{Methodology}

The study is aimed to finding a new way to limit the spread of the Coronavirus due to the threat to human life. It causes the death rate is increasing day by day throughout the globe. To add to this pandemic situation control, this study effort to achieve number of confirmed cases, recoveries and deaths in the upcoming 20 days. In the study, the dataset used holds regular time series, containing the number of confirmed cases, deaths, and recoveries in the past number of days from which the pandemic started until 4 October 2020.

We will predict the number of confirmed cases, recoveries and deaths. The first step is to process the data. In next step, the dataset (259) split into two subsets: a training set (220 days) and testing set (39 days). The ML models used in this paper are NN, SVM, BR and PR. These models trained on the days confirmed cases, recovery, and death patterns. The evaluating matrix consist of number of parameters such as MSE, RMSE, and MAE and $\mathrm{R}^{2}$. The proposed approach used in this article has been illustrated in Fig. 1 block diagram.

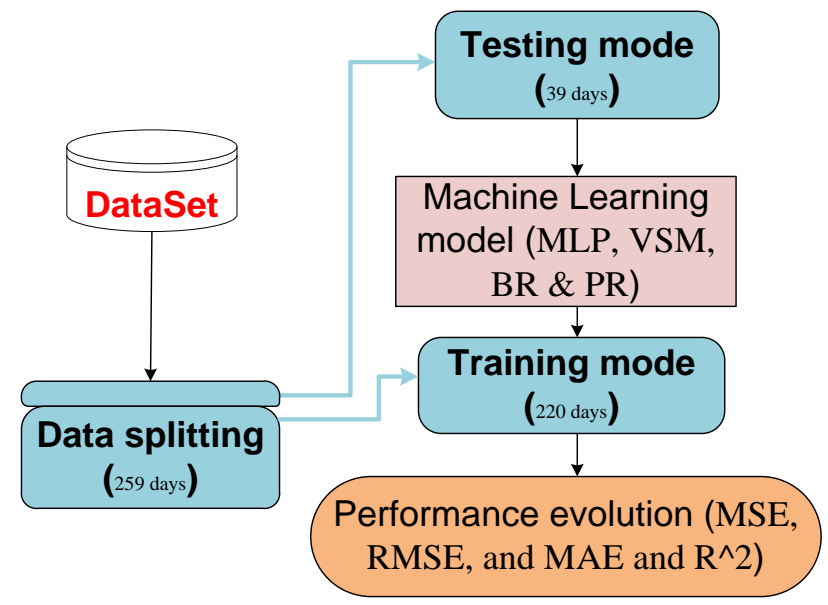

Fig.1. proposed approach

\section{A. Covid 19 Data Visualization}

All data until 4 October 2020 has been used to generate the prediction results as in Fig. 2,3,4 and 5 where the log figures are shown from Fig. 6 to Fig. 9. The world daily cases presented from Fig. 10 to Fig. 12.

The figures displayed the data on moving average for 10 days which can be calculate at time period $(t)$ as in equation:

$$
\frac{\left(x_{t}+x_{t-1}+x_{t-2}+. .+x_{M-(t-1)}\right)}{M}
$$

where $(\mathrm{M})$ represents the sliding window, it depends on the amount of smoothing desired since increasing the value of (M) improves the smoothing at the expense of accuracy. 


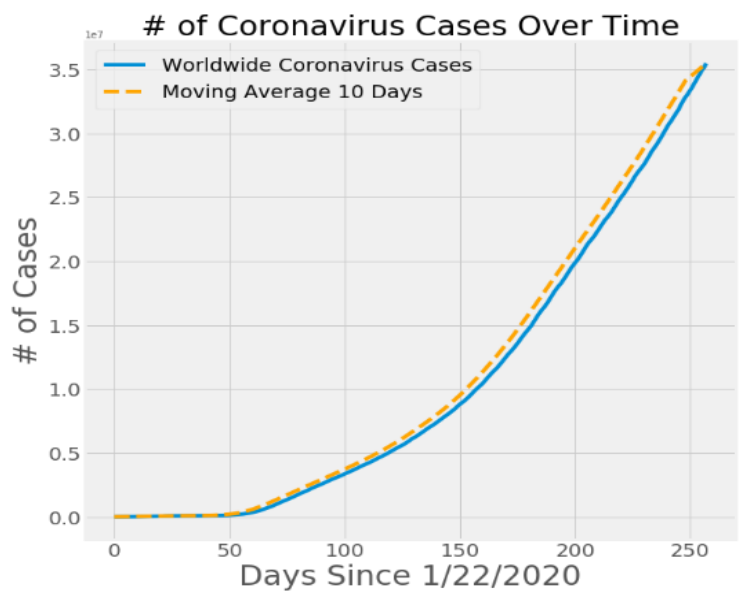

Fig.2. number of confirmed cases in worldwide

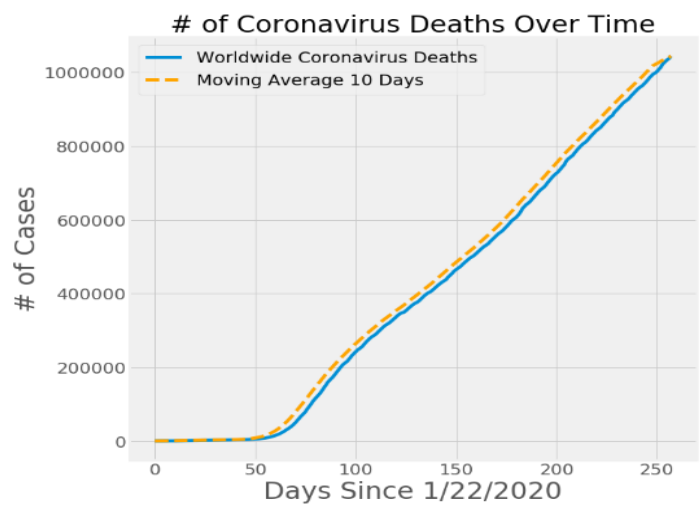

Fig.3. number of deaths cases in worldwide

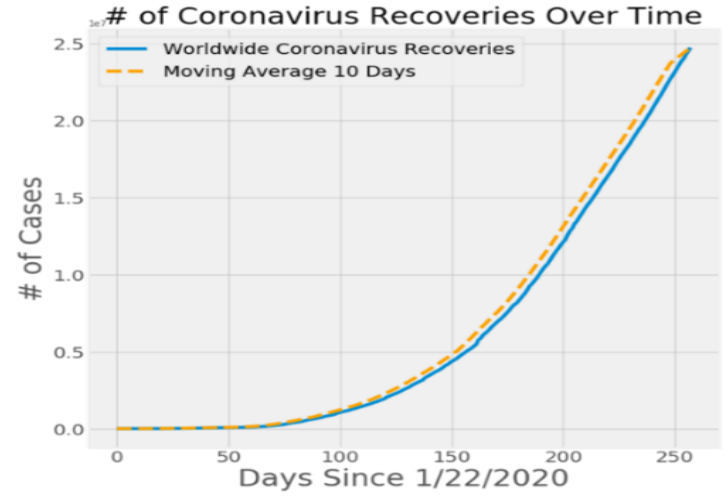

Fig.4. number of recoveries cases in worldwide

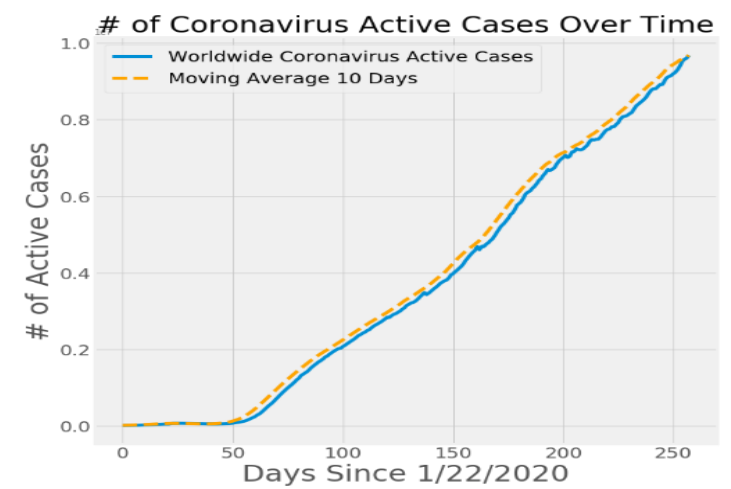

Fig.5. number of active cases in worldwide 


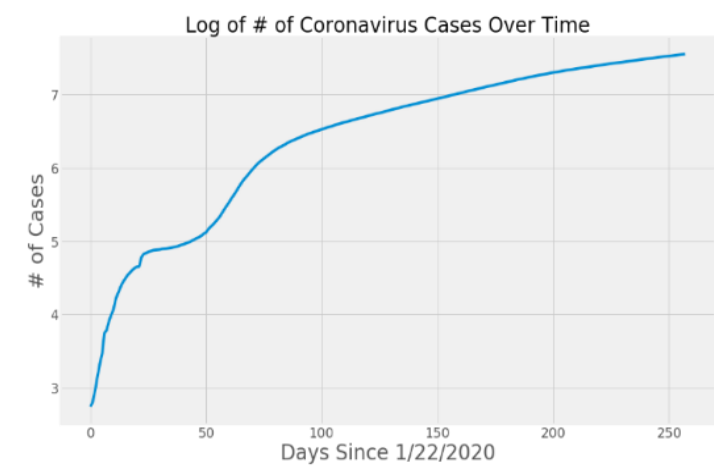

Fig.6. $\log$ of COVID 19 cases in worldwide

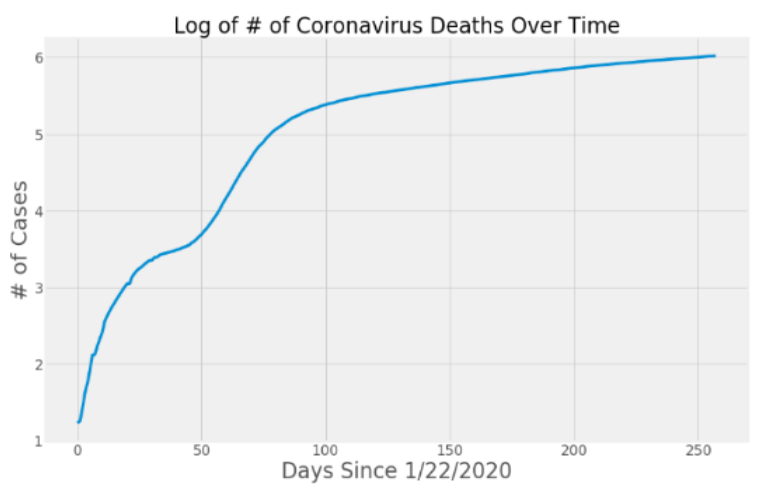

Fig.7. $\log$ of deaths cases in worldwide

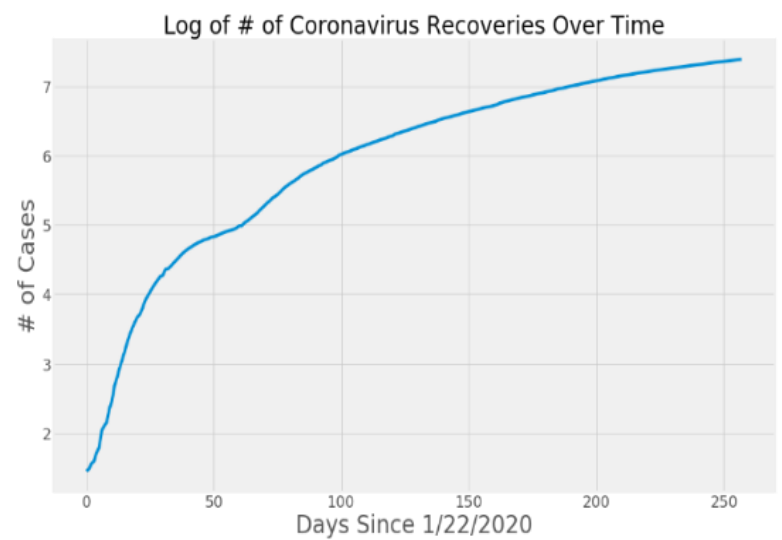

Fig.8. $\log$ of recoveries cases in worldwide

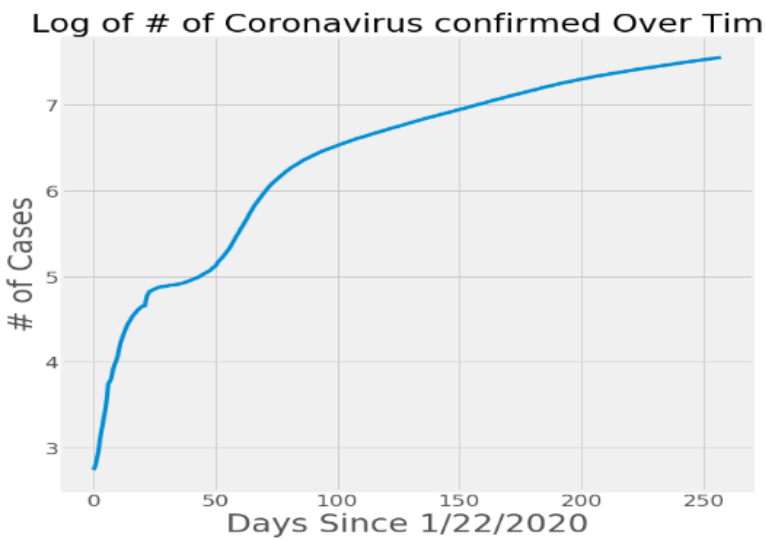

Fig.9. $\log$ of confirmed cases in worldwide 


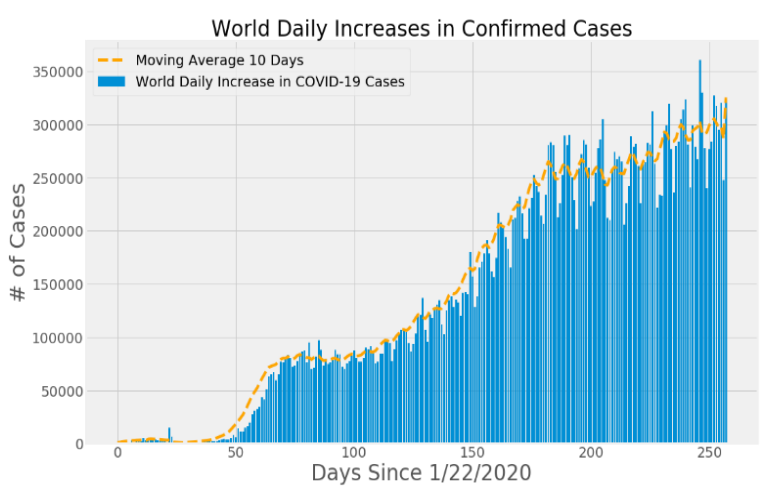

Fig.10. word daily increases in confirmed cases

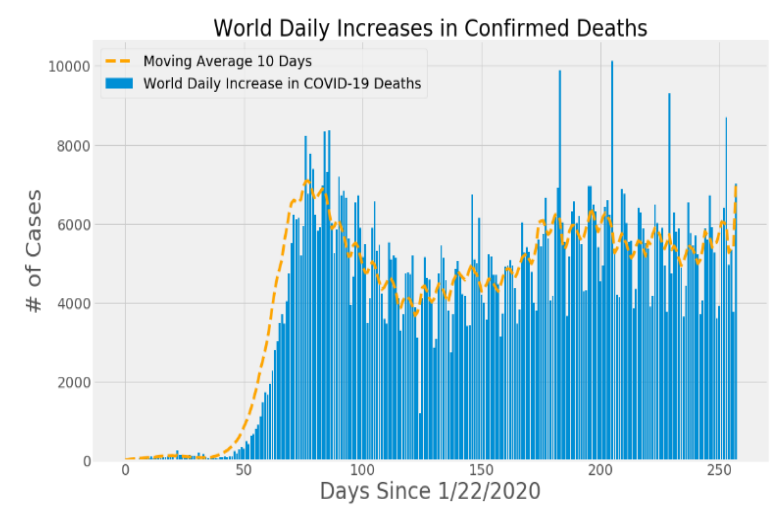

Fig.11. word daily increases in confirmed deaths cases

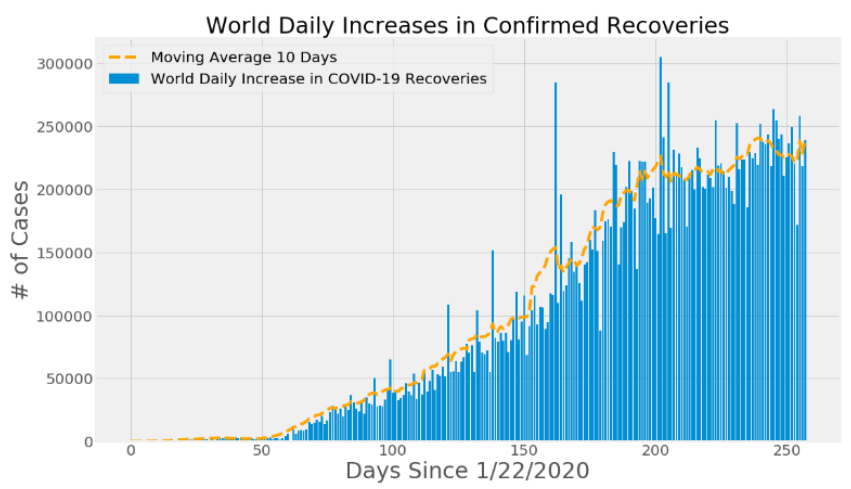

Fig.12. word daily increases in confirmed recoveries cases

\section{Results and Discussion:}

The number of people infected with COVID-19 is unknown in the world. In this paper, we are trying to know the numbers of infected cases and death cases, in addition to the cases of recovery affected by COVID 19 in the next 20 days. Four machine learning models MLP, PR, SVM, and BR have been used to forecast the number of confirmed cases, the deaths, and recoveries. Four measures have been used to evaluate the four algorithms and choose the best algorithm that predicts the number of COVID 19 cases.

\subsection{Prediction of New Confirmed Cases}

In the following, figures are presented the performance graphs of SVM, PR, MLP, BR and linear regression models respectively. All Graphs in all figures predict that the new confirmed case will increase in upcoming days which is a not good sign expect, the results of MLP give us the hopefulness. We can observe clearly from Fig. 18, the MLP curve (B), outperform other algorithms as in curve (A). 


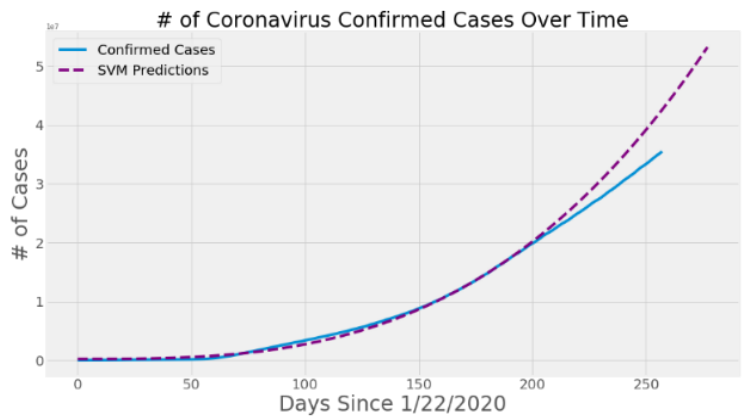

Fig.13. SVM prediction for the upcoming 20 days (New confirm cases).

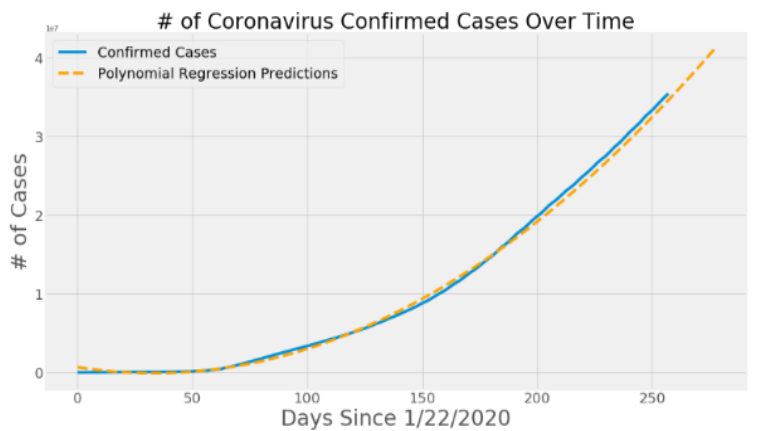

Fig.14. PR prediction by for the upcoming 20 days (New confirm cases).

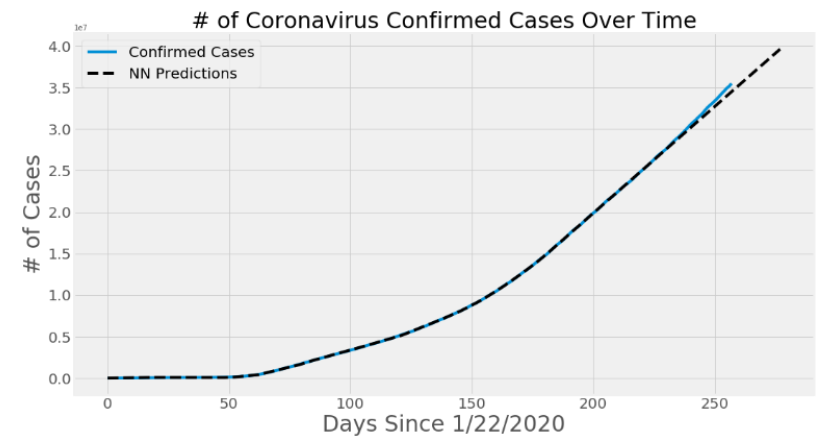

Fig.15. NN (i.e. MLP) prediction for the upcoming 20 days (New confirm cases).

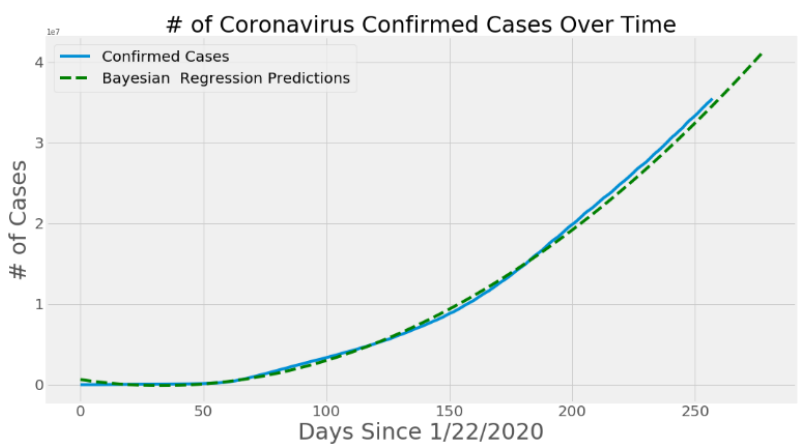

Fig.16. B. R. prediction for the upcoming 20 days (New confirm cases). 


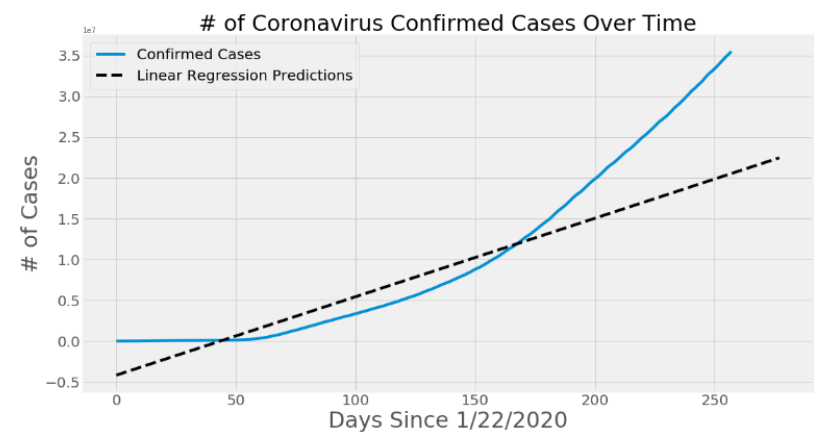

Fig.17. L.R prediction for the upcoming 20 days (New confirm cases)

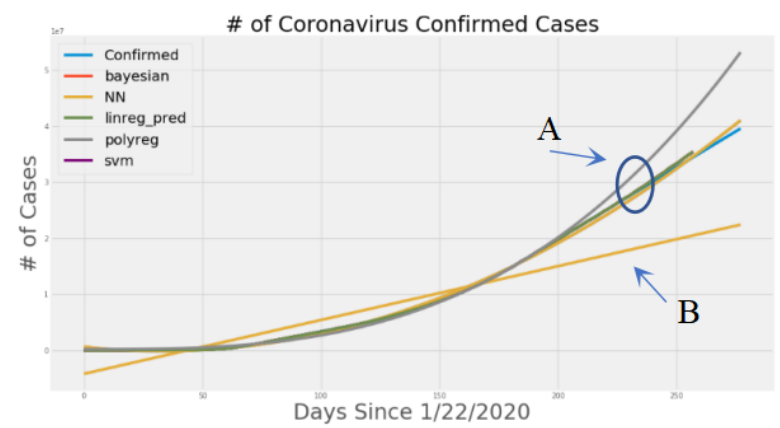

Fig.18. Prediction algorithm for the upcoming 20 days (New confirm cases).

The algorithm NN (i.e. MLP) achieved the best prediction performance for new cases as in curve (B) in Fig. 18. While the algorithm SVM was performing poorly. The performance of the algorithms PR and BR was good and comparable in performance. As for the linear algorithms, they were not able to predict the states and the values were very far from reality. The study performs predictions on confirmed case and according to results MLP performs better among all the models, while PR and BR perform equally well and achieve almost the same $\mathrm{R}^{2}$ as shown in Table 4 . The predicted number of confirmed cases over the worldwide are list in Table 5.

Table 4. Evaluate Metrics to Confirmed Cases Prediction

\begin{tabular}{|c|c|c|c|c|}
\hline & SVM & PR & $\mathrm{BR}$ & NN (i.e. MLP) \\
\hline MAE & 3350432.468 & 888352.8451 & 906398.9479 & 268264.6905 \\
\hline MSE & 14851309552451.30 & $7.90834 \mathrm{E}+11$ & $8.23477 \mathrm{E}+11$ & $1.62164 \mathrm{E}+11$ \\
\hline $\mathrm{R}^{\wedge} 2$ & 0.593436004 & 0.953576088 & 0.951513218 & 0.989086182 \\
\hline Explained Variance & 0.900738369 & 0.999902393 & 0.999887051 & 0.993298691 \\
\hline RMSE & 1830.418659 & 942.5247186 & 952.0498663 & 517.9427483 \\
\hline
\end{tabular}


Predicted \# of Confirmed Cases Worldwide

Table 5. Predicted of Confirmed Cases Worldwide

\begin{tabular}{|c|c|c|c|c|c|}
\hline & Date & $\begin{array}{l}\text { Polynomial Predicted \# of } \\
\text { Confirmed Cases Worldwide }\end{array}$ & $\begin{array}{l}\text { SVM Predicted \# of } \\
\text { Confirmed Cases } \\
\text { Worldwide }\end{array}$ & $\begin{array}{l}\text { Bayesian Predicted \# of } \\
\text { Confirmed Cases } \\
\text { Worldwide }\end{array}$ & $\begin{array}{l}\text { NN (i.e. MLP) \# of } \\
\text { Confirmed Cases } \\
\text { Worldwide }\end{array}$ \\
\hline 0 & $10 / 6 / 2020$ & 34925200 & 43049834 & 34895230 & 34755613 \\
\hline 1 & $10 / 7 / 2020$ & 35237460 & 43549854 & 35206971 & 35012449 \\
\hline 2 & $10 / 8 / 2020$ & 35551106 & 44053750 & 35520094 & 35269286 \\
\hline 3 & $10 / 9 / 2020$ & 358666137 & 44561537 & 35834599 & 35526123 \\
\hline 4 & $10 / 10 / 2020$ & 36182555 & 45073230 & 36150485 & 35782960 \\
\hline 5 & $10 / 11 / 2020$ & 36500357 & 45588844 & 36467754 & 36039796 \\
\hline 6 & $10 / 12 / 2020$ & 36819546 & 46108393 & 36786404 & 36296633 \\
\hline 7 & $10 / 13 / 2020$ & 37140120 & 46631894 & 37106436 & 36553470 \\
\hline 8 & $10 / 14 / 2020$ & 37462080 & 47159361 & 37427850 & 36810307 \\
\hline 9 & $10 / 15 / 2020$ & 37785426 & 47690809 & 37750646 & 37067143 \\
\hline 10 & $10 / 16 / 2020$ & 38110157 & 48226252 & 38074824 & 37323980 \\
\hline 11 & $10 / 17 / 2020$ & 38436274 & 48765706 & 38400383 & 37580817 \\
\hline 12 & $10 / 18 / 2020$ & 38763776 & 49309186 & 38727325 & 37837269 \\
\hline 13 & $10 / 19 / 2020$ & 39092665 & 49856707 & 39055648 & 38091178 \\
\hline 14 & $10 / 20 / 2020$ & 39422938 & 50408283 & 39385353 & 38345087 \\
\hline 15 & $10 / 21 / 2020$ & 39754598 & 50963930 & 39716440 & 38598996 \\
\hline 16 & $10 / 22 / 2020$ & 40087643 & 51523663 & 40048908 & 38852905 \\
\hline 17 & $10 / 23 / 2020$ & 40422074 & 52087496 & 40382759 & 39106814 \\
\hline 18 & $10 / 24 / 2020$ & 40757891 & 52655445 & 40717991 & 39360724 \\
\hline 19 & $10 / 25 / 2020$ & 41095093 & 53227524 & 41054605 & 39614633 \\
\hline
\end{tabular}

\subsection{Prediction of New Recoveries Cases}

The recovery cases of COVID-19 increase day by day, Graphs in figures 19, 20, 21, 22 and 23 are illustrates the predictions of learning models. Table 6 shows the estimating results to recovery cases of the models used in this study. Table 7 provides the predicted of recovered cases over the worldwide. We found that, the results also proved the superiority of MLP over the rest of the models, and both BR and PR were equal.

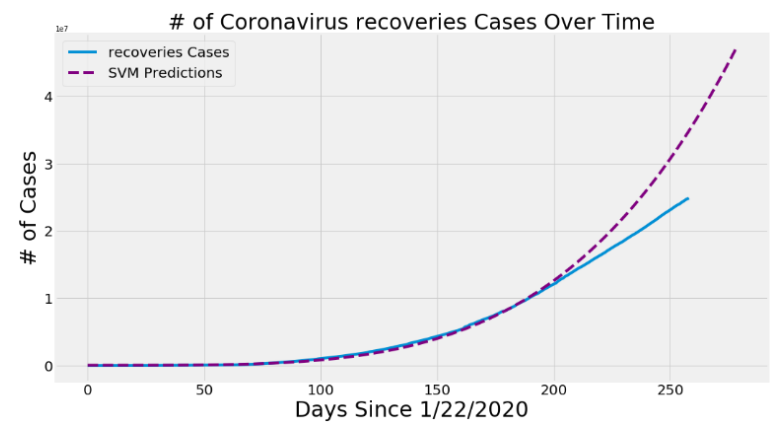

Fig.19. SVM prediction for the upcoming 20 days (New recoveries cases).

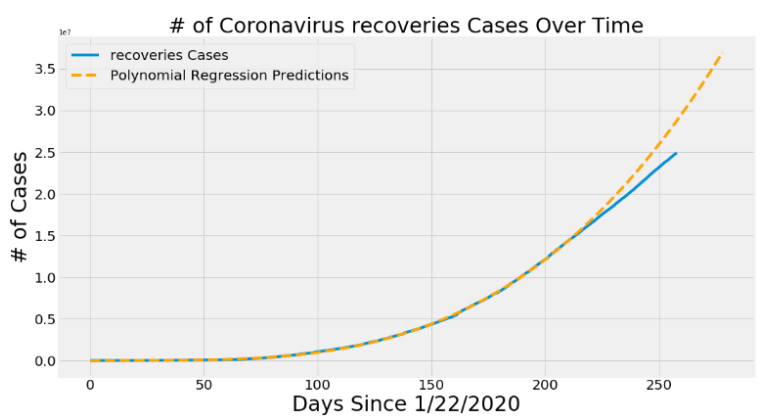

Fig.20. PR prediction for the upcoming 20 days (New recoveries cases) 


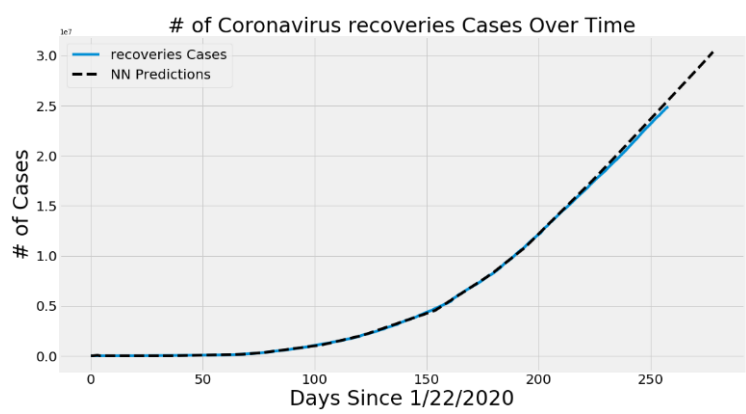

Fig.21. MLP prediction for the upcoming 20 days (New recoveries cases).

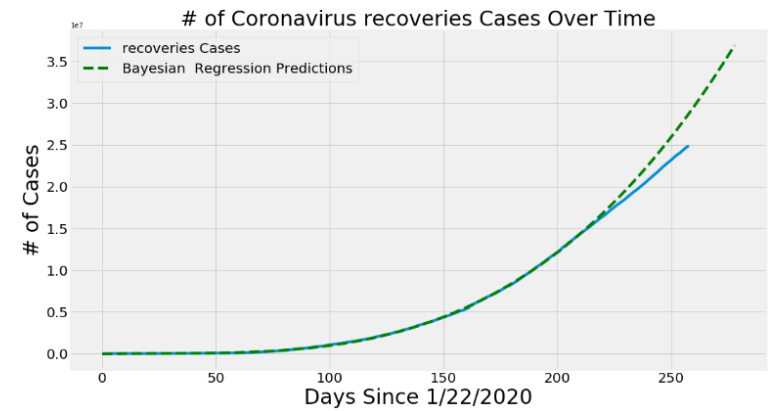

Fig.22. B.R. prediction for the upcoming 20 days (New recoveries cases).

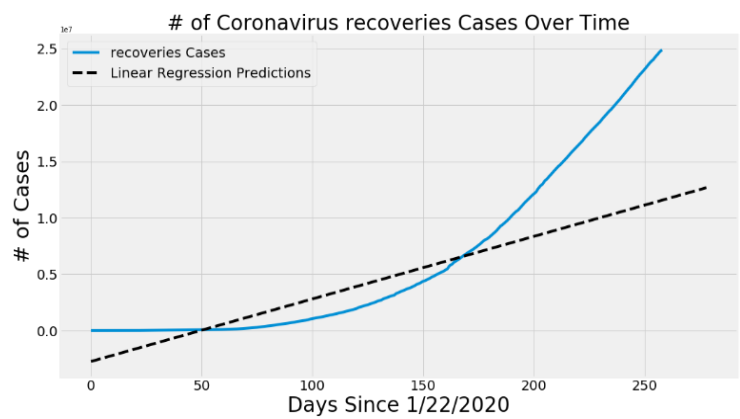

Fig.23. LR prediction by for the upcoming 20 days (New recoveries cases).

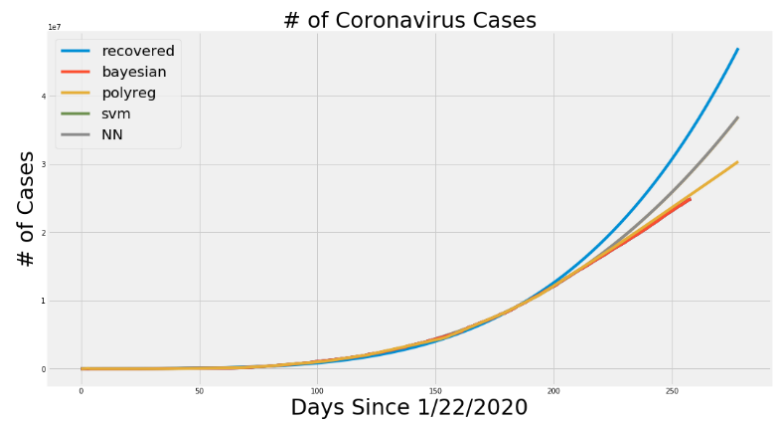

Fig.24 ML algorithm prediction by for the upcoming 20 days (New recoveries cases).

Table 6. Evaluate Metrics to recoveries Cases Prediction

\begin{tabular}{|c|c|c|c|c|}
\hline & SVM & PR & BR & NN (i.e. MLP) \\
\hline MAE & 4271162.927 & 1318443.424 & 1261822.89 & 246086.8444 \\
\hline MSE & 25161649191032.50 & $2.94424 \mathrm{E}+12$ & $2.72036 \mathrm{E}+12$ & $1.04288 \mathrm{E}+11$ \\
\hline $\mathbf{R}^{\wedge} \mathbf{2}$ & 0.286567242 & 0.849124653 & 0.85858648 & 0.989356735 \\
\hline Explained Variance & 0.803824055 & 0.937260783 & 0.939929732 & 0.995537118 \\
\hline RMSE & 2066.679203 & 1148.234917 & 1123.308902 & 496.0714106 \\
\hline
\end{tabular}


Predicted of recovered Cases Worldwide:

Table 7. Predicted of recovered Cases Worldwide

\begin{tabular}{|c|c|c|c|c|c|}
\hline & Date & $\begin{array}{l}\text { Polynomial Predicted \# of } \\
\text { recovered Cases } \\
\text { Worldwide }\end{array}$ & $\begin{array}{l}\text { SVM Predicted \# of } \\
\text { recovered Cases } \\
\text { Worldwide }\end{array}$ & $\begin{array}{c}\text { Bayesian Predicted \# } \\
\text { of recovered Cases } \\
\text { Worldwide }\end{array}$ & $\begin{array}{c}\text { Predicted \# NN (i.e. MLP) } \\
\text { of recovered Cases } \\
\text { Worldwide }\end{array}$ \\
\hline 0 & $10 / 7 / 2020$ & 29170602 & 35417364 & 29144438 & 25783933 \\
\hline 1 & $10 / 8 / 2020$ & 29549772 & 35966719 & 29522921 & 26022671 \\
\hline 2 & $10 / 9 / 2020$ & 29932228 & 36522449 & 29904679 & 26261409 \\
\hline 3 & $10 / 10 / 2020$ & 30317984 & 37084604 & 30289727 & 26500146 \\
\hline 4 & $10 / 11 / 2020$ & 30707055 & 37653233 & 30678078 & 26738884 \\
\hline 5 & $10 / 12 / 2020$ & 31099455 & 38228385 & 31069748 & 26977622 \\
\hline 6 & $10 / 13 / 2020$ & 31495198 & 38810110 & 31464749 & 27216360 \\
\hline 7 & $10 / 14 / 2020$ & 31894298 & 39398459 & 31863097 & 27455097 \\
\hline 8 & $10 / 15 / 2020$ & 32296769 & 39993480 & 32264805 & 27693835 \\
\hline 9 & $10 / 16 / 2020$ & 32702626 & 40595225 & 32669887 & 27932573 \\
\hline 10 & $10 / 17 / 2020$ & 33111883 & 41203743 & 33078359 & 28171310 \\
\hline 11 & $10 / 18 / 2020$ & 33524555 & 41819086 & 33490233 & 28410048 \\
\hline 12 & $10 / 19 / 2020$ & 33940654 & 42441304 & 33905523 & 28648786 \\
\hline 13 & $10 / 20 / 2020$ & 34360197 & 43070448 & 34324245 & 28889115 \\
\hline 14 & $10 / 21 / 2020$ & 34783196 & 43706570 & 34746413 & 29135897 \\
\hline 15 & $10 / 22 / 2020$ & 35209666 & 44349721 & 35172039 & 29382680 \\
\hline 16 & $10 / 23 / 2020$ & 35639621 & 44999952 & 35601140 & 29629462 \\
\hline 17 & $10 / 24 / 2020$ & 36073076 & 45657316 & 36033727 & 29876245 \\
\hline 18 & $10 / 25 / 2020$ & 36510044 & 46321863 & 36469817 & 30123027 \\
\hline 19 & $10 / 26 / 2020$ & 36950541 & 46993648 & 36909423 & 30369810 \\
\hline
\end{tabular}

\subsection{Prediction of New Deaths Cases}

The performance of the NN (i.e. MLP), PR, SVM, and BR models are illustrated in Fig. 25, 26, 27, 28 and 29 respectively. All figures are predicting that the death rate will be growing in upcoming days, which are a very worrying marking. Through this sign, new precautionary precautions must be taken or an algorithm for life must be created in a different way in order to come with this virus. From Table 8 we can observe that the MLP outperform other approach in $\mathrm{R}^{\wedge} 2$ parameter. The Predicted of Deaths Cases Worldwide provide in Table 9.

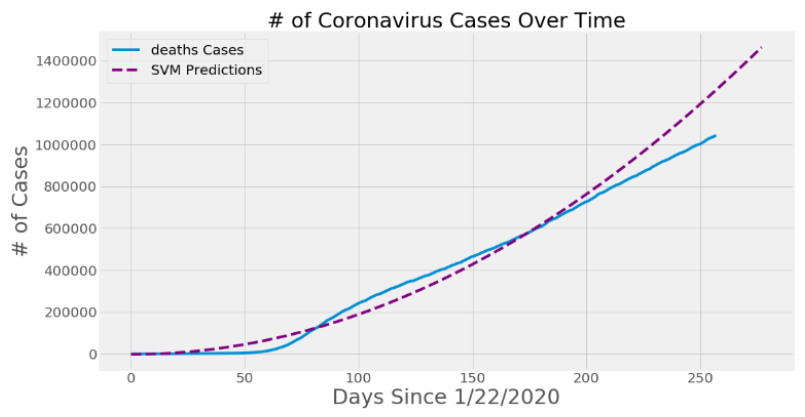

Fig.25. SVM prediction for the upcoming 20 days (New Deaths cases).

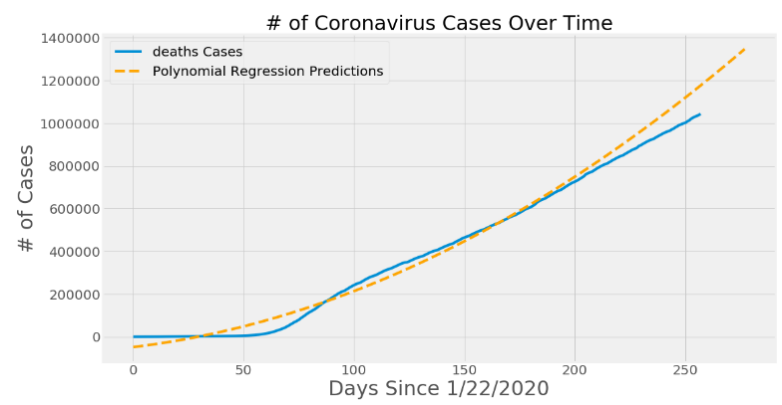

Fig..26. PR prediction for the upcoming 20 days (New Deaths cases). 


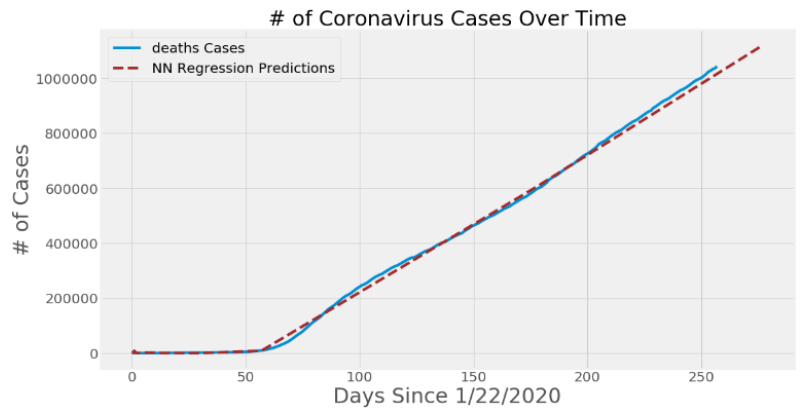

Fig.27. NN (i.e. MLP) prediction for the upcoming 20 days (New Deaths cases).

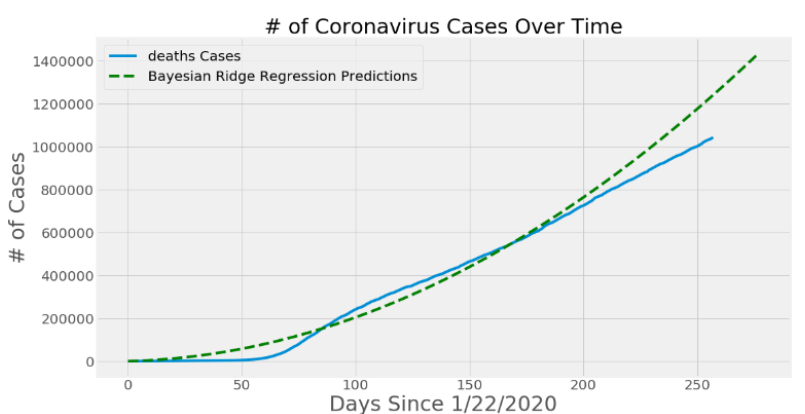

Fig.28. B.R. prediction for the upcoming 20 days (New Deaths cases).

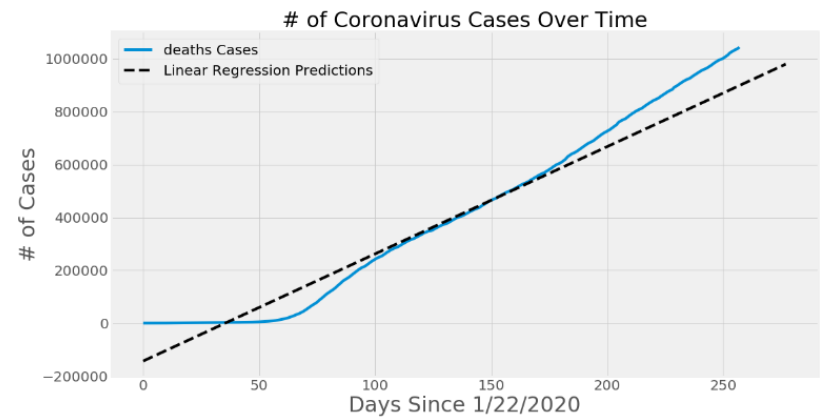

Fig.29. LR prediction for the upcoming 20 days (New Deaths cases).

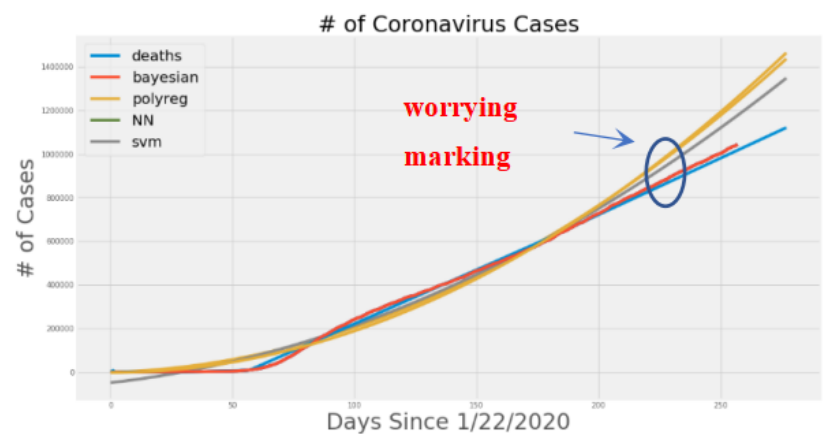

Fig.30. ML algorithm prediction for the upcoming 20 days (New Deaths cases).

Table 8. Evaluate Metrics to Deaths Cases Prediction

\begin{tabular}{|c|c|c|c|c|}
\hline & SVM & PR & BR & NN (i.e. MLP) \\
\hline MAE & 55870.28183 & 56511.30021 & 112902.4181 & 19754.56174 \\
\hline MSE & 4323906787.89 & 5930203438 & 14845929533 & 406564135.9 \\
\hline $\mathbf{R}^{\wedge} \mathbf{2}$ & 0.67934414 & 0.6711188 & 0.083891571 & 0.932880987 \\
\hline Explained Variance & 0.910830061 & 0.839648927 & 0.870477134 & 0.997305522 \\
\hline RMSE & 236.3689528 & 237.7210555 & 336.0095505 & 140.5509222 \\
\hline
\end{tabular}


Predicted \# of death Cases Worldwide

Table 9. Predicted of Deaths Cases Worldwide

\begin{tabular}{|c|c|c|c|c|c|}
\hline & Date & $\begin{array}{c}\text { Polynomial Predicted \# of } \\
\text { deaths Cases Worldwide }\end{array}$ & $\begin{array}{c}\text { SVM Predicted \# of deaths } \\
\text { Cases Worldwide }\end{array}$ & $\begin{array}{c}\text { Bayesian Predicted \# of } \\
\text { deaths Cases Worldwide }\end{array}$ & $\begin{array}{c}\text { NN Predicted \# of deaths } \\
\text { Cases Worldwide }\end{array}$ \\
\hline 0 & $10 / 6 / 2020$ & 1184576 & 1269107 & 1250318 & 1021946 \\
\hline 1 & $10 / 7 / 2020$ & 1192890 & 1278980 & 1259753 & 1027132 \\
\hline 2 & $10 / 8 / 2020$ & 1201231 & 1288890 & 1269224 & 1037503 \\
\hline 3 & $10 / 9 / 2020$ & 1209600 & 1298839 & 1278730 & 1042689 \\
\hline 4 & $10 / 10 / 2020$ & 1217996 & 1308825 & 1288272 & 1047875 \\
\hline 5 & $10 / 11 / 2020$ & 1226419 & 1318850 & 1297849 & 1053060 \\
\hline 6 & $10 / 12 / 2020$ & 1234870 & 1328914 & 1307462 & 1063436 \\
\hline 7 & $10 / 13 / 2020$ & 124347 & 1331710 & 1068618 \\
\hline 8 & $10 / 14 / 2020$ & 1251853 & 1349155 & 1073803 \\
\hline 9 & $10 / 15 / 2020$ & 1260385 & 1359332 & 1326794 & 1078989 \\
\hline 10 & $10 / 16 / 2020$ & 1268945 & 1369548 & 1346513 & 1084175 \\
\hline 11 & $10 / 17 / 2020$ & 1277532 & 1379802 & 1356057 & 1089361 \\
\hline 12 & $10 / 18 / 2020$ & 1286146 & 1390095 & 1365882 & 1094546 \\
\hline 13 & $10 / 19 / 2020$ & 1294788 & 1400425 & 1375743 & 1099732 \\
\hline 14 & $10 / 20 / 2020$ & 1303457 & 1410794 & 1385639 & 1104918 \\
\hline 15 & $10 / 21 / 2020$ & 1312153 & 1421201 & 1395571 & 1110103 \\
\hline 16 & $10 / 22 / 2020$ & 1320877 & 1431646 & 1405538 & 1115289 \\
\hline 17 & $10 / 23 / 2020$ & 1329627 & 1442129 & 1415540 & 1120475 \\
\hline 18 & $10 / 24 / 2020$ & 1338406 & 1452651 & 1425578 & \\
\hline 19 & $10 / 25 / 2020$ & 1347211 & 1463210 & 1435652 & \\
\hline
\end{tabular}

\subsection{Impact of Model Performances with 20 Days Prediction Intervals}

To ensure the accuracy and reliability of the results, our model predictions are very auspicious that show in Fig. 31, 32 and 33, because the models predict that in upcoming days' death rate will be increased and the scheme of mortality rate shows the same pattern. Furthermore, in recovery scenario models predict that recoveries rate will be also increase and in Fig. 32 the recovery scheme follows the same behavior which proves the model predictions correct.

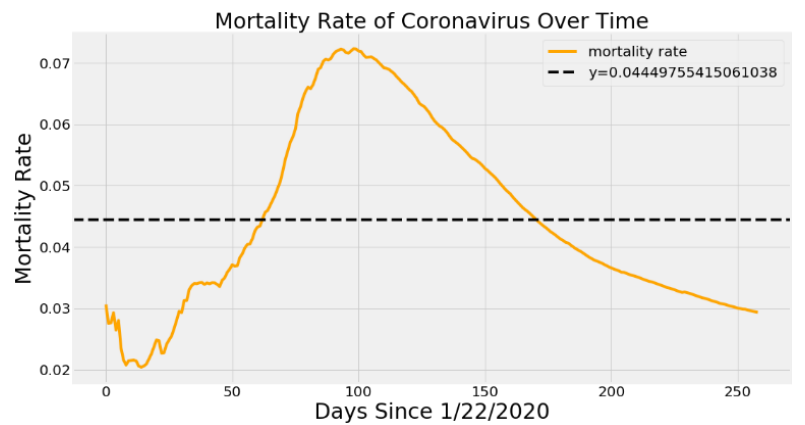

Fig.31. Mortality rate after 20 days of this paper prediction

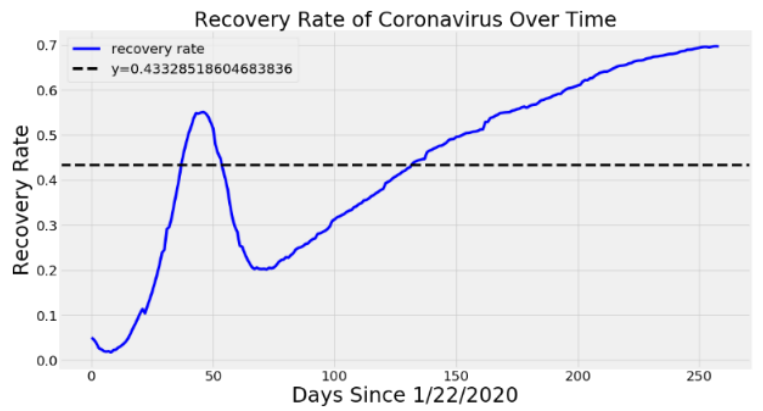

Fig..32. Recovery rate after 20 days of this paper prediction. 


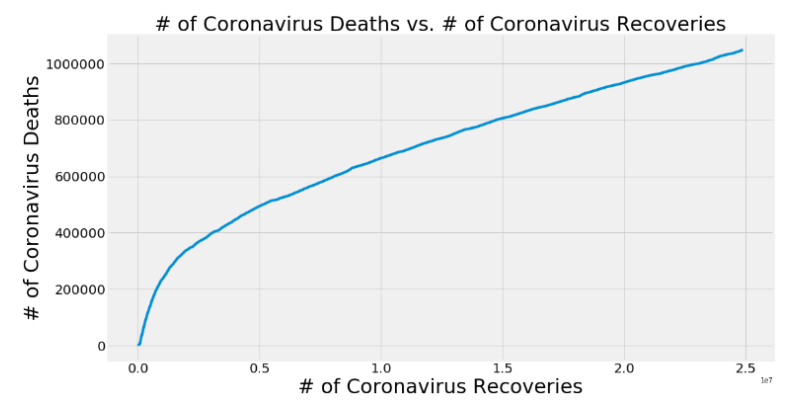

Fig.33. Ratio between recovery rate and death rate after 20 days of this paper prediction

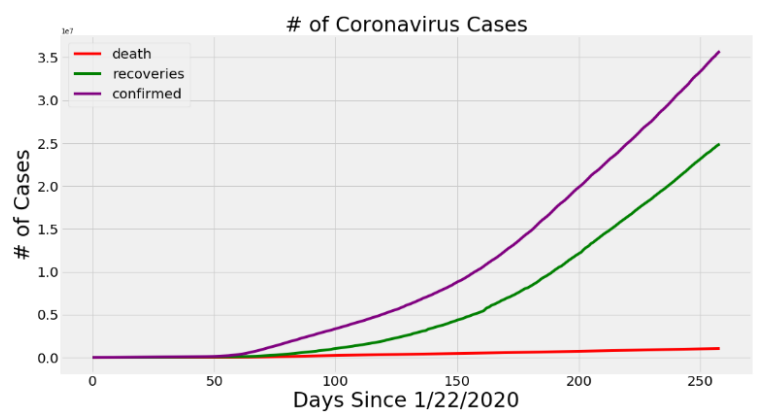

Fig..34. Comparison between death rate, recovery rate and confirm case rate after 5 days of this study prediction

In general, MPL performed best followed by VSM performed followed by PR and then BR and the death has lower values compare to new and recovery case. From Fig. 34, more stringent restrictions must be imposed by governments and the World Health Organization to limit the spread of the Coronavirus.

\section{Conclusion}

The Coronavirus has caused great economic losses resulting from the imposition of strict measures to confront this virus, including a complete or partial curfew, and the closure of airports and factories, as well as the virus, has caused great loss of life. Scientists and governments fear the second wave of this virus. In this paper, we are building a system to predict the spread of the Coronavirus in the world based on machine learning algorithms. Corona data provided by Johns Hopkins University have been analyzed and predict the spread of the Coronavirus in the coming days to help control this virus. The results showed that smart neural networks outperformed all other algorithms and achieved amazing results with a low error rate. The results showed that injuries will increase slightly in the coming days, and our result deploy the optimism because the deaths will increase. This study will benefit governments in understanding the general situation and taking appropriate measures. The results prove that MLP performs best in the current forecasting domain given the nature and size of the dataset. All algorithms also perform well for forecasting, to some extent, to predict the death rate as in Fig. 30 observe that the situation going to worry marking.

According to the results of all approach, the death rates will increase in upcoming days, and recoveries rate will be slowed down. The poor results clearly have been observed in SVM scenarios because of the ups and downs in the dataset values. This is due to the difficulty of establishing accurate patterns between the given values of the data set. Overall we conclude that model predictions according to the current scenario are correct which may be helpful to understand the upcoming situation.

It is clear that there is a visible effect to the whole world because the Corona virus does not spread to the same extent that it has spread in some countries, due to the exposure of those countries to ultraviolet rays with a value greater than 10 and I think there are other factors. Therefore, the study must be developed to include all natural factors such as relative humidity, solar ultra-violate (UV), erythema dose, UV aerosol index, day light and ozone thick layer.

\section{Acknowledgment}

We would like to express our appreciation to all those who have supported us during our research and study in the Faculty of Engineering at Mashreq University (Sudan).

\section{References}

[1] C. Sohrabi et al., "World Health Organization declares global emergency: A review of the 2019 novel coronavirus (COVID19)," 2020. 
[2] S. K. Mohanty et al., "Severe acute respiratory syndrome coronavirus-2 (SARS-CoV-2) and coronavirus disease 19 (COVID19)-anatomic pathology perspective on current knowledge," vol. 15, no. 1, pp. 1-17, 2020.

[3] A. Rajkomar, J. Dean, and I. J. N. E. J. o. M. Kohane, "Machine learning in medicine," vol. 380, no. 14, pp. 1347-1358, 2019.

[4] A. Jakaria, M. M. Hossain, and M. A. J. a. p. a. Rahman, "Smart weather forecasting using machine learning: a case study in tennessee," 2020.

[5] D. S. Hain and R. Jurowetzki, "The promises of Machine Learning and Big Data in entrepreneurship research," in Handbook of quantitative research methods in entrepreneurship: Edward Elgar Publishing, 2020.

[6] M. Arttürk, S. Yavuz, and T. J. M. M. i. I. S. Allahviranloo, "Artificial Intelligence and Autonomous Car," pp. 391-412, 2020.

[7] Smriti Ayushi, V R Badri Prasad, " Cross-Domain Recommendation Model based on Hybrid Approach", International Journal of Modern Education and Computer Science(IJMECS), Vol.10, No.11, pp. 36-42, 2018.DOI: 10.5815/ijmecs.2018.11.05

[8] B. Nazlı, Y. Gültepe, and H. Altural, "Classification of Coronary Artery Disease Using Different Machine Learning Algorithms," 2020.

[9] G. Battineni, G. G. Sagaro, N. Chinatalapudi, and F. J. J. o. P. M. Amenta, "Applications of Machine Learning Predictive Models in the Chronic Disease Diagnosis," vol. 10, no. 2, p. 21, 2020.

[10] M. Maniruzzaman, M. J. Rahman, B. Ahammed, M. M. J. H. I. S. Abedin, and Systems, "Classification and prediction of diabetes disease using machine learning paradigm," vol. 8, no. 1, p. 7, 2020.

[11] S. Barik, S. Mohanty, D. Rout, S. Mohanty, A. K. Patra, and A. K. Mishra, "Heart Disease Prediction Using Machine Learning Techniques," in Advances in Electrical Control and Signal Systems: Springer, 2020, pp. 879-888.

[12] R. Y. Kumbhar and S. J. S. i. I. P. N. Vijaykumar, "Breast Cancer Prediction Using Machine Learning Algorithm," vol. 40, no. 68, pp. 380-395, 2020.

[13] Q. Li et al., "Early transmission dynamics in Wuhan, China, of novel coronavirus-infected pneumonia," 2020.

[14] Mirza Waseem Hussain, Tabasum Mirza, Malik Mubasher Hassan. " Impact of COVID-19 Pandemic on the Human Behavior ", International Journal of Education and Management Engineering (IJEME), Vol.10, No.5, pp.35-61, 2020. DOI: 10.5815/ijeme.2020.05.05

[15] M. P. Kelly, "Digital technologies and disease prevention," American journal of preventive medicine, vol. 51, no. 5, pp. 861$863,2016$.

[16] S. Mahmoud, M. Hussein, and A. Keshk, "Predicting Future Products Rate using Machine Learning Algorithms," International Journal of Intelligent Systems \& Applications, vol. 12, no. 5, 2020.

[17] J. H. U. D. Repository. (2020, 4/OTC). COVID-19 Data. Available: https://github.com/CSSEGISandData/COVID-19

[18] T. M. Mitchell, "Machine Learning, volume 1 of 1," ed: McGraw-Hill Science/Engineering/-Math, 1997.

[19] G. E. J. M. L. P. Hinton and M. P. Methods", "Connectionist learning procedures. Artificial Intelligence, 40 1-3: 185 234, 1989. Reprinted in J. Carbonell, editor,"" 1990.

[20] X. Glorot and Y. Bengio, "Understanding the difficulty of training deep feedforward neural networks," in Proceedings of the thirteenth international conference on artificial intelligence and statistics, 2010, pp. 249-256.

[21] K. He, X. Zhang, S. Ren, and J. Sun, "Delving deep into rectifiers: Surpassing human-level performance on imagenet classification," in Proceedings of the IEEE international conference on computer vision, 2015, pp. 1026-1034.

[22] D. P. Kingma and J. J. a. p. a. Ba, "Adam: A method for stochastic optimization," 2014.

[23] C. Cortes and V. Vapnik, "Support-vector networks," Machine Learning, vol. 20, no. 3, pp. 273-297, 1995/09/01 1995.

[24] D. J. J. N. c. MacKay, "Bayesian interpolation," vol. 4, no. 3, pp. 415-447, 1992.

[25] M. E. J. J. o. m. 1. r. Tipping, "Sparse Bayesian learning and the relevance vector machine," vol. 1, no. Jun, pp. 211-244, 2001.

[26] H. Huang, M. J. A. A. Abdel-Aty, and Prevention, "Multilevel data and Bayesian analysis in traffic safety," vol. 42, no. 6, pp. $1556-1565,2010$.

\section{Authors' Profiles}

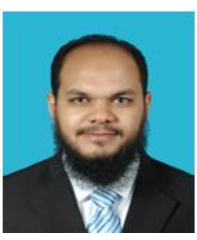

Ahmed Hassan M. Hassan: Associate Professor for Communication Engineering at Mashreq University, Sudan. His research interesting in Wireless Networks, and Machine Learning.

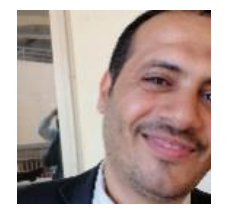

Arfan Ali Mohammed Qasem: Lecturer in Mechatronics Engineering at Mashreq University. His research interesting in Machine Learning, Machine Learning, AI, Image Processing and Analysis. 


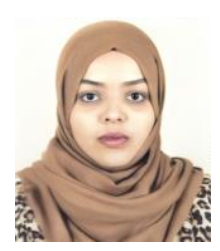

Walaa Faisal Mohammed Abdalla: Lecturer in Telecommunication Engineering department, Faculty of Engineering at Mashreq University. Her research interesting in in 5G wireless network, Antenna and wave propagation and Machine Leaning.

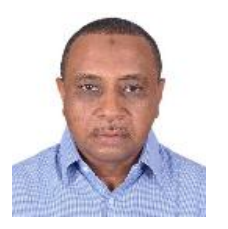

Omer H. Elhassan: President of Mashreq University, major of computer science, Artificial Intelligent and Data Mining.

How to cite this paper: Ahmed Hassan Mohammed Hassan, Arfan Ali Mohammed Qasem, Walaa Faisal Mohammed Abdalla, Omer H. Elhassan, "Visualization \& Prediction of COVID-19 Future Outbreak by Using Machine Learning", International Journal of Information Technology and Computer Science(IJITCS), Vol.13, No.3, pp.16-32, 2021. DOI: 10.5815/ijitcs.2021.03.02 